\title{
Der SSRI verstärkt den Effekt
}

Fragestellung: Verstärkt die Kombination mit Escitalopram die Effekte einer internetbasierten kognitiven Verhaltenstherapie (IKVT) bei sozialer Phobie und verändert sie die Reaktivität und Konnektivität des Gehirns?

Hintergrund: Bei sozialen Phobien werden selektive SerotoninReuptake-Inhibitoren (SSRI) und kognitive Verhaltenstherapie (KVT) im klinischen Alltag oft zeitgleich angewandt, jedoch wurde bisher in nur wenigen Studien der Effekt der Kombination erforscht, auch im Hinblick auf einen Zusammenhang zwischen sozialer Phobie und einer Hyperaktivität der Amygdala.

Patienten und Methodik: In der placebokontrollierten Doppelblindstudie mit Neuroimaging wurden 48 Teilnehmer mit der Hauptdiagnose „soziale Phobie“ nach DSM-IV als Einschlusskriterium randomisiert in zwei Behandlungsgruppen eingeteilt. Ausschlusskriterien waren Kontraindikationen für ein MRT, schwere somatische Erkrankungen, gravierende psychiatrische Störungen, Behandlung jeglicher psychiatrischer Störung innerhalb der letzten drei Monate, Menopause sowie Drogen- oder Alkoholmissbrauch. Ergänzend zur IKVT-Behandlung erhielt eine Gruppe zusätzlich $20 \mathrm{mg} / \mathrm{Tag}$ Escitalopram, die andere Placebo über neun Wochen. Die IKVT war in neun wöchentliche Module aufgebaut, mit zusätzlichen Hausaufgaben. Ein

Gingnell M, Frick A, Engman J et al. Combining escitalopram and cognitive-behavioural therapy for social anxiety disorder: randomised controlled fMRI trial. Brit J Psychiatry 2016; 209: $229-35$ schriftliches Feedback erfolgte durch einen Therapeuten. Hauptendpunkte waren Symptomschwere auf der LSAS (Liebowitz Social Anxiety Scale) sowie das Ansprechen auf die Behandlung anhand der CGI-I (Clinical Global Impression - Improvement Scale). Mit letzterer erfolgte eine Bewertung als Responder oder Nonresponder. Zudem wurde die Erwartungsangst vor einem öffentlichen Vortrag mittels STAI-S (Spielberger State-Trait Anxiety Inventory - State Version) erfasst. Die Reaktivität des Gehirns wurde mit funktionaler MRT (fMRT) mit Aufgaben zum „Emotional Face-Matching“ bestimmt. Nach 15 Monaten erfolgte ein Followup mit der LSAS-Self-Report-Version. Die Adhärenz wurde durch die Bestimmung von Metaboliten im Blut erfasst.

Ergebnisse: In der Gruppe Escitalopram plus IKVT gab es signifikant $(\mathrm{p}=0,04)$ mehr Responder $(16 / 24)$ als in der Gruppe Placebo plus IKVT (8/24). Mittels STAI-S (Pre- zu Post-Treatment) konnte bezüglich der Erwartungsangst vor öffentlichen Vorträgen eine signifikante $(p=0,04)$ Gruppe $x$ Zeit-Interaktion zugunsten Escitalopram plus IKVT festgestellt werden, bei der LSAS ergab sich nur ein Trend $(p=0,09)$ zugunsten Escitalopram plus IKVT. Langfristig (Pre-Treatment zu Follow-up) jedoch zeigte sich hier eine signifikante $(p=0,02)$ Gruppe $x$ ZeitInteraktion zugunsten von Escitalopram plus IKVT.

Im fMRT (Pre- zu Post-Treatment) zeigte sich bei den Respondern im Vergleich zu den Nonrespondern eine signifikant ( $\mathrm{p}$ fwe $=0,019$ ) stärkere Verminderung der Reaktivität der Amygdala. Verbesserungen der LSAS-Werte korrelierten mit einer verminderten Reaktivität der rechten Amygdala.

Schlussfolgerungen: Eine Kombination mit Escitalopram verstärkt den Effekt der IKVT bei der Behandlung sozialer Phobien. Ein Behandlungserfolg geht mit einer Verminderung der Reaktivität der Amygdala einher, jedoch scheint letzteres kein pharmakodynamischer Effekt des Escitaloprams zu sein, sondern mit der Befundbesserung zusammenzuhängen.

\section{- Kommentar von Michael Fuchs, Erlangen}

\section{Positive Effekte mit weiterem Forschungsbedarf}

Die Studie, welche mit einem Jadad-Score von 5 Punkten hohe methodische Ansprüche erfüllt, legt nahe, dass die im klinischen Alltag oft angewandte Kombination von SSRI (hier Escitalopram) zusätzlich zur KVT sinnvoll ist, um sowohl die Wahrscheinlichkeit eines Ansprechens auf die Therapie zu erhöhen als auch langfristig bessere Ergebnisse zu erzielen. Ein Kritikpunkt an dieser Studie ist allerdings die eher geringe Probandenzahl, gerade was Vergleiche zwischen Respondern und Nonrespondern jeweils innerhalb einer Gruppe anbelangt. Auch der fehlende direkte Vergleich zu Monotherapien sollte angeführt werden, die Autoren verweisen hier lediglich auf mögliche Vergleiche mit Studien, die sie im Vorfeld durchgeführt hatten. Im Hinblick auf die klinische Relevanz wären für die Zukunft auch vergleichbare Studien mit zusätzlichem Augenmerk auf häufige Komorbiditäten wie zum Beispiel Depressionen oder Alkoholmissbrauch wünschenswert. Nichts- destotrotz stützen die vorliegenden Ergebnisse eine im klinischen Alltag bereits gängige Kombinationstherapie bei sozialer Phobie, die das Outcome auch nach Beendigung der medikamentösen Therapie langfristig positiv beeinflusst.

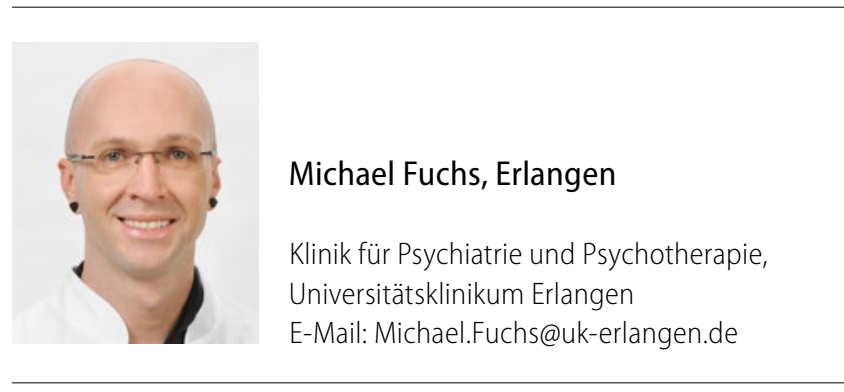

